$\xi=-1$

\title{
Dyeability of Red Cabbage Colorant on Silk Fabric Treated With Entada Spiralis Extracts
}

\author{
N. Mat Shom ${ }^{1}$, M.I.A. Kadir ${ }^{2}$, M.R. Ahmad ${ }^{3 *}$ \\ Textile Research Group, Faculty of Applied Sciences, Universiti Teknologi MARA, 40450 Shah Alam Selangor, Malaysia \\ *Corresponding author E-mail: rozitex@salam.uitm.edu.my
}

\begin{abstract}
Of late, dyeing fabrics with natural colorants have been the attention of the industry. Natural colorants especially from plants have the advantage of being friendly towards the environment as the extraction, processing and dyeing procedures are considered green and safe. However, most natural dyes have poor colourfastness and needed mordants to improve their dyeability. In this study, the addition of Entada spiralis was utilized as a surface modifier to improve dyeability on $100 \%$ silk fabrics using extracts from red cabbage. The plant serves as a surface-active agent to reduce the surface tension of the fabrics. The surfactant is also a natural resource and therefore is not harmful to the environment. The Entada spiralis was extracted through boiling for 30 minutes on a hot plate before treating the extracts on the fabrics. During dyeing, lime was used as the mordant simultaneously with dyeing. The dyed fabrics were evaluated for colorfastness to washing, rubbing/crocking and perspiration using the Gray Scale Standard. In addition, the dyed fabrics were also measured for their colour strength, colour coordinates and \% reflectance. The results showed that dyed fabrics with Entada spiralis have darker shades in comparisons with the untreated fabric.
\end{abstract}

Keywords: Entada spiralis, natural dyes, red cabbage, silk, surfactant

\section{Introduction}

Synthetic dyes have been the dominant material for dyeing of textiles. The dyes can be easily obtained from the market and available in a wide range of colours with excellent overall colourfastness. Nevertheless, synthetic dyes are harmful to the environment. They are made from chemicals, relatively costly, toxic, not biodegradable and can cause health effects such as skin irritation [1]. Some researchers have identified that most azo-based synthetic dyes are harmful towards the ecology and human health as these dyes produce toxic chemicals $[2,3]$. As an alternative, natural dyes have gained a lot of interests as most of the processing methods do not contain harmful chemicals.

Natural colours can be obtained from plants, insects, minerals and have been used since ancient time for textile coloration $[4,5]$. Natural dyes produce uncommon, soft shades that are soothing to the eyes [6] which have that calm feeling and harmony with nature [7]. Saxena [7] also added that not only natural dyes are biodegradable, the residual vegetal matter after the extraction process could easily be composed or used as fertilizers. Besides the conventional boiling water extraction, natural dyes have been extracted using alternative methods such as using gamma, ultraviolet (UV), microwave and ultrasonic machines that may be able to save energy with low-temperature processing $[8,9]$. Researchers have also found that certain natural dyes have unique properties such as antimicrobial [10,11,12], insect repellent [13], deodorizing effects [14], and UV-protection [15, 16].

In this study, red cabbage or also known as Brassica oleracea $L$. was used as the natural dye source while Entada spiralis was used as a natural surfactant to treat the fabric samples before the dyeing process. According to Bruno et al. [17], pure extracts from red cabbage have shown to have antimicrobial effect under ambient conditions and can prevent microbial activity against Staphylococcus aureus pathogen. In addition, red cabbage is stable when exposed to temperature or UV radiation. A study by Ben Ticha [18] indicated that the dyeability of natural fabrics with colorants extracted from red cabbage can be improved by applying some cationic groups on the fiber. Hence, Entada spiralis was proposed in this study. Entada spiralis, also known locally as "beluru" or "sintok", a tree that grows freely and abundantly in Malaysia. The tree barks have many benefits and have been used as a shampoo, soap and also relieving treatment such as insect bites [19]. This plant can also be an antimicrobial and antifungal agent that can prevent from any fungal pathogens [20]. According to Olajide [21], Entada spiralis could be used to treat diseases such as bronchitis, coughs, miscarriage, and fever. The stem bark has saponin in the treatment of scalp which can avoid bacteria and skin epidermis. Mordants are used to fix the dye on the fabric effectively. Different types of mordants will give different colour shades on the fabric. Baliarsingh [22] and Saxena [7] stated that the effectiveness of mordant on colour has been proven to give good wash fastness as the mordant forms an insoluble complex with the dye.

\section{Experimental}

\subsection{Materials}

The study used $100 \%$ plain silk fabric while lime was used as the mordant during dyeing. Red cabbage was purchased from a local market. The barks of Entada spiralis (Figure 1) were obtained from local sources in the state of Kelantan, Malaysia. 


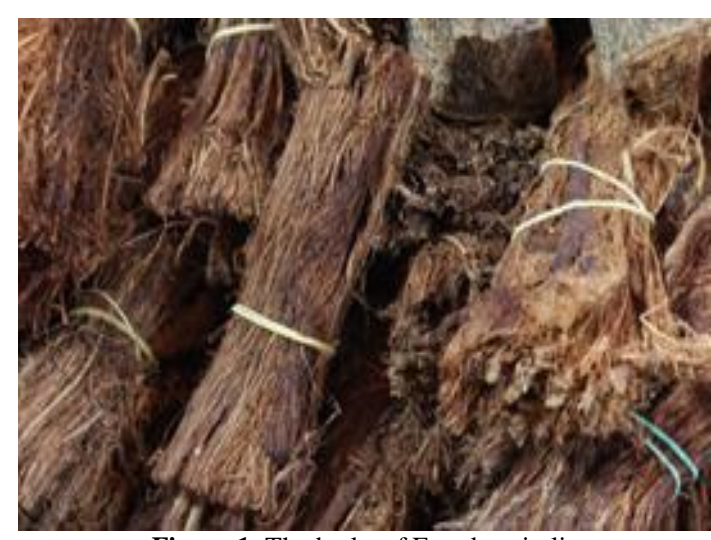

Figure 1: The barks of Entada spiralis

\subsubsection{Natural dye extraction}

The boiling water extraction method was used to extract the colours from the red cabbage. The red cabbage was first chopped into small pieces and then grinded using a dry blender into much smaller sizes. In the extraction process, a liquor ratio of 1:20 was used. The Entada spiralis plant was also extracted through the same method and with the same liquor ratio. The extracted mixtures were then cooled down and sieved before using them to dye the fabric.

\subsubsection{Dyeing and mordanting}

Exhaust dyeing was performed onto the silk fabrics using the red cabbage extracted colorants. Before the dyeing process, the fabrics were treated with Entada spiralis, acting as a surfactant to enhance the surface of the fabric. The dyeing process took 60 minutes at $80^{\circ} \mathrm{C}$ with a liquor ratio of 1:20. Meta-mordanting method was used where dyeing and mordanting, using lime, were done simultaneously. The use of mordant was about $2 \%$ of the weight of the fabric. After dyeing and mordanting, the samples were squeezed, rinsed and dried.

\subsubsection{Colour Measurements}

The shades of the dyed fabrics were measured using HunterLab LabScan XE spectrophotometer and analysed using EasyMatch QC software. The results are given as $\mathrm{L}^{*}, \mathrm{a}^{*}, \mathrm{~b}^{*}$ which are the colour coordinates. The $\mathrm{L}^{*}$ values gives the lightness or darkness in which a 0 value indicates black while a 100 value indicates white. As for the $a^{*}$ values, the $(+a)$ values indicates redness while $(-a)$ values indicate greenness. As for $b^{*}$, the $(+b)$ shows yellowness while the (-b) values shows blueness. In addition, the colour strength $(\mathrm{K} / \mathrm{S})$ and percent reflectance values were also given by the spectrophotometer. The K/S values were calculated using the Kubelka-Monk equation (Equation 1) to determine the dye uptake for the sample which was at maximum absorption wavelength from the reflectance value.

$$
\frac{K}{S}=\frac{1-0.01 R^{2}}{2(0.01) R}
$$

The dyed fabrics were also measured for their colourfastness properties towards washing, rubbing/crocking (in dry and wet condition), perspiration and light fastness in accordance to the respective standard test methods (Malaysian Standards, MS ISO). The changes in colour were observed using a standard Gray Scale Rating under a D65 CIE Standard Illuminant light inside a Colour Matching cabinet. Figure 2 and 3 show the lightfastness machine and crockmeter that was used to test the dyed silk fabric. Table 1 shows the standard methods for colour assessments.

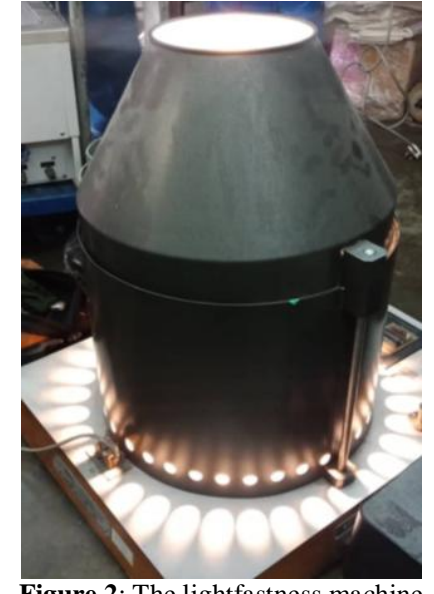

Figure 2: The lightfastness machine

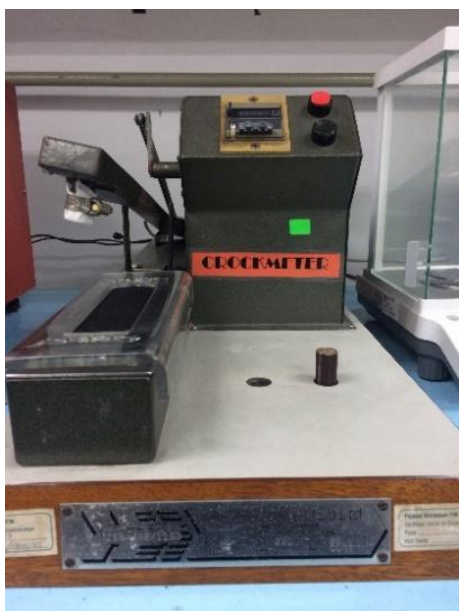

Figure 3: The crockmeter machine for rubbing/crocking test

Table 1: The Colorfastness Assessments by using Standard Methods

\begin{tabular}{|l|l|l|}
\hline Colourfastness & Standard Methods & Equipments \\
\hline Perspiration & MS ISO 105 - E04-1996 & Perspirometer \\
& MS ISO 105 - A05 - 2003 & Change in color \\
& MS ISO 105 - A04 - 2003 & Staining \\
\hline Washing & MS ISO 105 - C01 - 1996 & Auto wash \\
& MS ISO 105 - A05 - 2003 & Change in color \\
& MS ISO 105 - A04 - 2003 & Staining \\
\hline Light & MS ISO 105-B02-2001 & Light \\
& Light & Fastness \\
& & Tester \\
\hline Crocking/ & MS ISO 105 - X12-2001 & Crockmeter \\
Rubbing & MS ISO 105 - A04 - 2003 & Staining \\
\hline
\end{tabular}

\section{Results and Discussion}

\subsection{Shades of the dyed samples}

An example of the colour shades obtained after dyeing for both untreated and treated samples are shown in Table 2. It can be seen that the sample treated with Entada spiralis prior to dyeing have a darker shade in comparisons with the untreated sample. This result was supported by the colour coordinates or the $\mathrm{L}^{*} \mathrm{a}^{*} \mathrm{~b}^{*}$ values given in Table 3 and illustrated in Figure 4.

It can be seen from Table 2 that the untreated sample have somewhat lower L*, a*, b* values than the samples treated with Entada spilaris. The treated sample is darker (lower $\mathrm{L}^{*}$ ) and is somewhat more reddish and brownish in comparisons with the untreated sample. It can be seen the treated sample has 78.36 value in reflectance which is much lower than the untreated sample. The treated sample with lower $\mathrm{L}^{*}$ value gave darker colour shade. The untreated sample has a 83.93 value in reflectance. A higher $\mathrm{L}^{*}$ value indicated light shades of colour. Basically, the treated samples 
with Entada spiralis as the surfactant contributed to give much darker colour shades to the dyed fabrics.

Table 2: Shades of the dyed fabrics

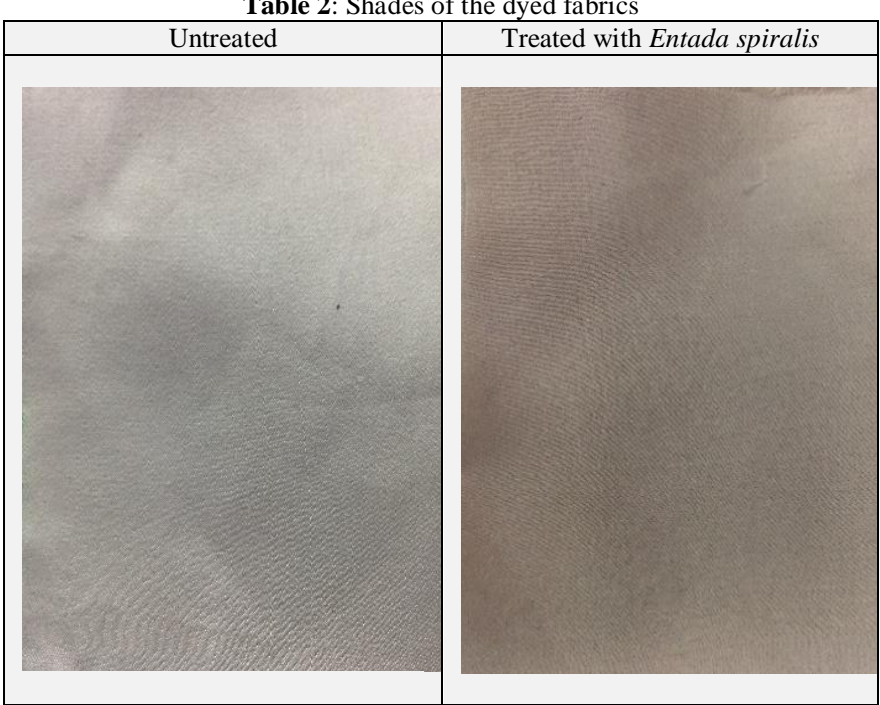

Table 3: $\mathrm{L}^{*} \mathrm{a} * \mathrm{~b} *$ values of the samples

\begin{tabular}{|c|c|c|c|c|c|c|c|}
\hline & $\mathrm{L}^{*}$ & $\mathrm{a} *$ & $\mathrm{~b}^{*}$ & $\mathrm{dL}^{*}$ & $\mathrm{da}^{*}$ & $\mathrm{db}^{*}$ & $\mathrm{~K} / \mathrm{S}$ \\
\hline $\begin{array}{c}\text { Control } \\
\text { sample }\end{array}$ & 90.69 & 2.40 & -6.26 & 90.69 & 2.40 & -6.26 & 0.65 \\
\hline $\begin{array}{c}\text { Untreated } \\
\text { sample }\end{array}$ & 83.93 & 3.85 & -2.93 & -6.76 & 1.45 & 3.33 & 0.70 \\
\hline $\begin{array}{c}\text { Treated } \\
\text { sample }\end{array}$ & 78.36 & 5.28 & 1.90 & -12.33 & 2.87 & 8.16 & 0.87 \\
\hline
\end{tabular}

From Table 3, it can be seen that the $\mathrm{L}^{*}$ value for the control (undyed) sample is 90.69 while the $\mathrm{a}^{*}$ value is 2.40 and $\mathrm{b}^{*}$ value, 6.26. The differences between the untreated sample and undyed sample $\left(\mathrm{dL}^{*}\right)$ is -6.76 . This means that the untreated sample has a slight darker shade than the control sample, which was due to the red cabbage dye. The $+\mathrm{a}$ value indicates reddish colour. The untreated sample has a value of 3.85 for $\mathrm{a}^{*}$, and the differences between the untreated and undyed samples is 1.45 . The $-\mathrm{b}$ indicates blueish colour. The $b^{*}$ value for the untreated sample is -2.93 . The $\mathrm{db}^{*}$ value is 3.33 , which means that the untreated sample's colour is more to reddish and yellowish compared to the control sample. The differences between the control sample and treated sample is 12.33. This data showed the treated sample has a darker colour shade than the undyed and untreated samples. The $\mathrm{L}^{*}$ value for the treated sample has the lowest reflectance value compared to other samples. The differences of the $\mathrm{a}^{*}$ value for the undyed and treated sample is 2.87. This indicates that the sample is more reddish in colour compared to the untreated sample. The differences in the $b^{*}$ value for the undyed and treated sample is 8.16. The treated sample has a darker shade in yellowish colour compared to the untreated sample. The Entada spiralis gave the dyed fabrics a darker colour shade compared to samples without Entada spiralis as the surfactant. The coordinates for $\mathrm{a}^{*} \mathrm{~b}^{*}$ are plotted in 2D plots as shown in Figure 4. In the figure, number 1 is the treated sample while number 2 is the untreated sample.

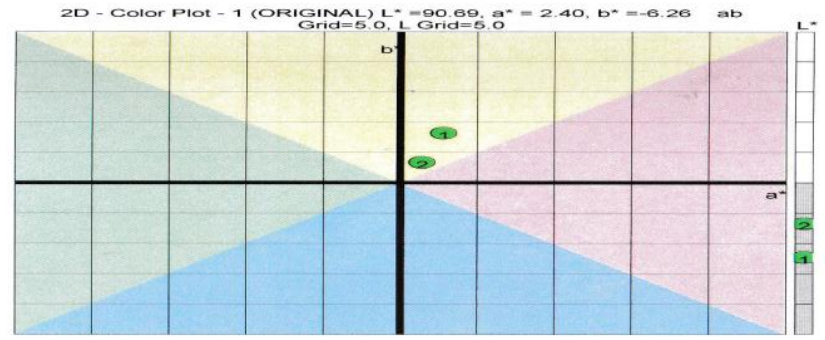

Figure 4: 2D Plot representing the shades of the untreated and treated samples

\subsection{Colour Strength}

The colour strength $(\mathrm{K} / \mathrm{S})$ and $\%$ reflectance curves are shown in Figures 5 and 6, respectively. It can be seen that all the dyed samples showed similar curves because they came from the same sources, red cabbage and Entada spiralis. The K/S value for the treated sample is higher than the untreated sample. The K/S value for the treated sample is 0.87 while the untreated sample is 0.70 . This clearly shows that the treated samples has a higher reflectance and is less absorbance. While for the untreated sample, it has a lower K/S value due to the higher absorbance and lower reflectance values. In general, the treated fabrics gave higher K/S value with the presence of Entada spiralis as a surfactant. It is understood that when the reflectance is more, the absorbance is less and when reflectance is less, the absorbance is more. Color strength $(\mathrm{K} / \mathrm{S})$ is one of the most important parameter to test the quality measurement of a sample in terms of depth of the color dyed fabric. Figures 7 and 8 show the K/S value at the minimum wavelength and $\%$ reflectance.

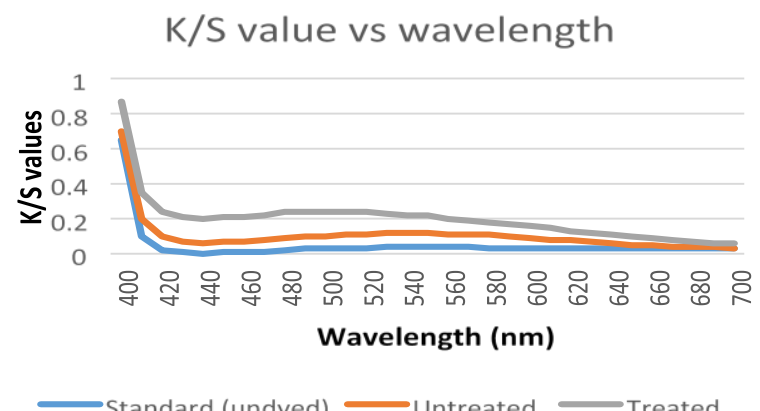

Figure 5: K/S Chart for the dyed samples

\section{$\%$ Reflectance wavelength}

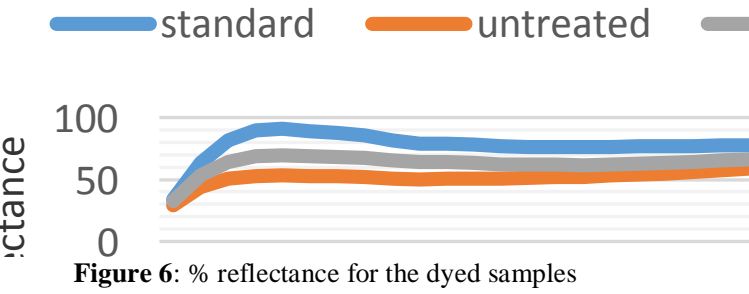

\section{K/S VALUE AT MINIMUM WAVELENGTH}

E Standard (Undyed) E Untreated

1

0.8

0.6

0.4

0.2

0

$\mathrm{K} / \mathrm{S}$ value at minimum wavelength

Figure 7: K/S chart at the minimum wavelength 


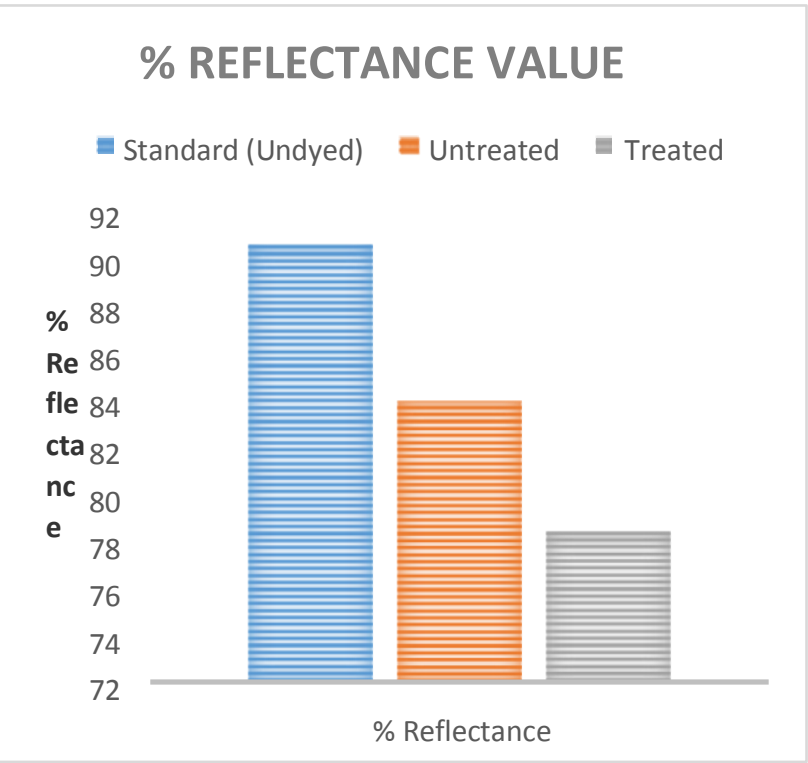

Figure 8: \% Reflectance chart treated sample.

\subsection{Colourfastness properties}

Table 4 shows the results of colourfastness properties from the dyed silk fabrics. For wash fastness properties, the untreated sample's rating is $3 / 4$ for change in colour but the treated sample gave a rating of $4 / 5$ which is considered as having good colourfastness. The untreated sample gave moderate colourfastness which indicates some colour fading after washing. The staining on the silk and cotton fabrics for the treated sample is rated at $4 / 5$ indicating having slight staining on the fabric. The staining for the untreated sample on the silk and cotton fabric also was rated at 4/5 which similarly indicates slight staining on the fabric. The fastness for perspiration was rated as having good colourfastness for both samples as they had a 4/5 rating. For staining, the cotton and silk fabric also gave $4 / 5$ rate which is good staining properties on the fabric. This shows that it has good colourfastness on perspiration. For rubbing fastness, in dry and wet conditions, both samples were rated at 5 which show excellent colourfastness to rubbing. Unfortunately, the light fastness properties results were rated at 2 indicating poor colourfastness for both samples as they were found to fade when exposed to light. Table 5 shows the changes in colour of the fabrics after the colourfastness assessment.

Table 4: The results for colorfastness properties

\begin{tabular}{|lll|c|c|}
\hline Test & & & Untreated sample & Treated sample \\
\hline Washing & & & $3 / 4$ & $4 / 5$ \\
& - & Silk & $4 / 5$ & $4 / 5$ \\
& - & Cotton & $4 / 5$ & $4 / 5$ \\
\hline Perspiration & & & $4 / 5$ & $4 / 5$ \\
& - & Silk & $4 / 5$ & $4 / 5$ \\
& - & Cotton & $4 / 5$ & $4 / 5$ \\
\hline Rubbing & & & 5 & 5 \\
& - & & 5 & 5 \\
\hline Light & - & Dry Wet & 2 & 2 \\
\hline
\end{tabular}

Table 5: The colourfastness result samples

\begin{tabular}{|l|l|l|}
\hline Test & Untreated sample & Treated sample \\
\hline Washing & & \\
& & \\
& & \\
\hline Perspiration & & \\
\hline
\end{tabular}

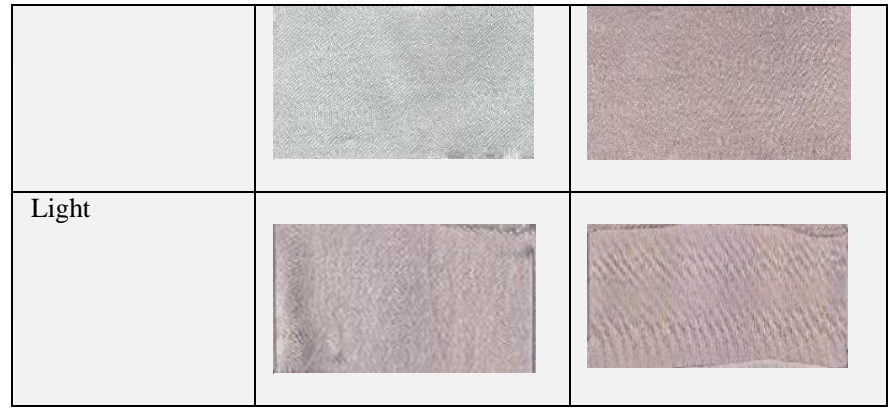

\section{Conclusion}

From the study, it can be concluded that silk fabrics can be dyed with natural dyes and surfactant to give darker colour shades than on untreated fabrics. The barks of Entada spiralis acted as a surfactant to fix the colour of the red cabbage on the silk fabric giving dark shades from light reddish to dark reddish. For the colour strength, higher reflectance was observed to be given by the untreated sample. Higher reflectance means lighter colour shades while lower reflectance gives darker colour shades. Fabric samples that were treated with Entada spiralis gave darker colour shades. The higher K/S values indicate higher colour strength and gave darker colour shades on the silk fabric. This was confirmed with the lower $\mathrm{L}^{*}$ values. For the treated fabric samples, good colourfastness were obtained for washing, perspiration and rubbing/crocking but somehow gave poor colorfastness in the light fastness test.

\section{Acknowledgement}

The authors would like to acknowledge the assistance given by the Research Management Center (RMC) of Universiti Teknologi MARA towards completing this project.

\section{References}

[1] Yang P, Shi W, Wang H \& Liu H (2016). Screening of freshwater fungi for decolorizing multiple synthetic dyes. Brazilian Journal of Mircobiology, 47(4), 828-834

[2] Islam SLJ, Shahid RM, Khan MA \& Mohammad F (2014). Study the effect of ammonia post-treatment on color characteristics of annatto-dyed textile substrate using reflectance spectrophotometery. Industrial Crops and Products 59, 337-342.

[3] Melgoza RM, Cruz A \& Buitron G (2004). Anaerobic/Aerobic treatment of colorants present in textile effluents. Water Science and Technology 5, 149-155.

[4] Shahid M, Islam S \& Mohammad F (2013). Recent advancements in natural dye applications: A review. Journal of Cleaner Production 53, 310-331.

[5] Yusuf M, Shahid M, Khan MI, Khan SA, Manzoor N \& Mohammad F (2015). Dyeing studies with henna and madder: A research on effect of tin (II) chloride mordant. Journal of Saudi Chemical Society $19,64-72$.

[6] Mongkholrattanasit R \& Krystufek J (2011). Dyeing, fastness, and UV protection properties of silk and wool fabrics dyed with eucalyptus leaf extract by the exhaustion process. Fibres Text East Eur, 19 (3), 94-99.

[7] Saxena S \& Raja ASM (2015). Roadmap to Sustainable Textiles and Clothing. Textile Science and Clothing Technology, 37-80.

[8] Adeel S, Hanif R, Zuber M \& Muneer M (2014). Ecofriendly dyeing of UV-irradiated cotton using extracts of Acacia nilotica bark (Kikar) as source of quercetin. Asian Journal of Chemistry 26, 830.

[9] Islam S \& Mohammad F (2015). High-energy radiation induced sustainable coloration and functional finishing of textile materials. Industrial \& Engineering Chemistry Research 54, 3727-3745.

[10] Shahid M, Ahmad A, Yusuf M, Khan MI, Khan SA, Manzoor N \& Mohammad F (2012). Dyeing, fastness and antimicrobial properties of woolen yarns dyed with gallnut (Quercus infectoria Oliv.) extract. Dyes and Pigments 95, 53-61. 
[11] Singh R, Jain A, Panwar S, Gupta D \& Khare SK (2005). Antimicrobial activity of some natural dyes. Dyes and Pigments 66, 99102.

[12] Yusuf M, Ahmad A, Shahid M, Khan MI, Khan SA, Manzoor N \& Mohammad F (2012). Assessment of colorimetric, antibacterial and antifungal properties of woollen yarn dyed with the extract of the leaves of henna (Lawsonia inermis). Journal of Cleaner Production 27, 42-50.

[13] Sricharussin W, Sopajaree C, Maneerung T \& Sangsuriya N (2009). Microencapsulated citronella oil for mosquito repellent finishing of cotton textiles. Journal of Texile 100, 682.

[14] Lee Y, Hwang E \& Kim H (2009). Colorimetric assay and antibacterial activity of cotton, silk and wool fabrics dyed with peony, pomegranate, clove, Coptis chinenis and gallnut extracts. Materials $2,10-21$.

[15] Grifoni D, Bacci L, Zipoli G, Carreras G, Baronti S \& Sabatini F (2009). Laboratory and outdoor assessment of UV protection offered by Flax and Hemp fabrics dyed with natural dyes. Photochemistry and Photobiology 85, 313-320.

[16] Sun SS \& Tang RC (2011). Adsorption and UV protection properties of the extract from Honeysuckle onto Wool. Industrial and Engineering Chemistry Research 50, 4217-4224.

[17] Bruno E, Campañone LA \& Martino M (2012). Some functional properties of pigment extracts from red cabbage (Brassica Oleracea) And Redbeet (Beta Vulgaris). Latin American Applied Research, 42, 427-432.

[18] BenTicha M, Haddar W, Meksi N \& Guesmi A (2016). Improving dyeability of modified cotton fabrics by the natural aqueous extract from red cabbage using ultrasonic energy. Carbohydrate Polymers 154, 287-295.

[19] Harun A, Mat So'ad SZ, Mohd Hassan N \& Che Mohd Ramli NK (2011). In vitro study of antifungal activity of Entada spiralis Ridl. crude extract against dermatophytes of superficial skin disease. Revelation and Science, (1), 57-61.

[20] Aboaba SS (2006). Antibacterial Effect of Edible Plant Extract on Escherichia coli 0157:H7. Pakistan Journal of Nutrition, 5(4), 325327.

[21] Olajide OA (2001). Studies on the antiinflammatory. Fitoterapia, 72, 492-496.

[22] Baliarsingh S, Behera PC, Jena J, Das T \& Das NB (2015). UV reflectance attributed direct correlation to colour strength and absorbence of natural dyed yarn with respect to mordant use and their potential antimicrobial efficacy. Journal of Cleaner Production 102, 485-492. 